GRÜNe,L.; WiRTh,F.

\title{
Stabilization of discrete-time bilinear systems
}

The problem of feedback stabilizing a semi-linear discrete-time system is studied. Under an accessibility condition on an associated nonlinear system on the projective space it can be shown that null controllability is equivalent to feedback stabilizability. We present a way in which a stabilizing feedback may be computed using some ideas from discounted optimal control.

\section{Introduction}

We consider systems on $\mathbb{R}^{d}$ of the form

$$
x(t+1)=A(u(t)) x(t):=\left(A_{0}+\sum_{i=1}^{m} u_{i}(t) A_{i}\right) x(t), \quad t \in \mathbb{N},
$$

where $A_{0}, \ldots, A_{m} \in \mathbb{R}^{d \times d}$ do not span a subspace of non-invertible matrices, and the set of admissible control values $U=\operatorname{cl} \operatorname{int} U \subset \mathbb{R}^{m}$ is compact, with connected interior and satisfies $0 \in \operatorname{int} U$. The associated system on the projective space $\mathbb{P}^{d-1}$ is given by

$$
\xi(t+1)=\mathbb{P} A(u(t)) \xi(t), \quad t \in \mathbb{N} .
$$

Here only those control sequences $u \in U^{\mathbb{N}}$ are admissible for which the corresponding solution $\Phi_{u}(t, 0) x_{0} \neq 0$ for all $t \in \mathbb{N}$, where $x_{0}$ is such that it spans $\xi_{0}$. These control values or sequences will be denoted by $U(\xi), U^{\mathbb{N}}(\xi)$. The solution of (2) corresponding to an initial value $\xi$ and a control sequence $u$ is denoted by $\varphi(\cdot, \xi, u)$. Let us note that the setup is a particular case of the systems studied in [3], [4]. Proofs for the statements of the theorems below can be found in [3].

We call system (1) asymptotically null-controllable if for every $x \in \mathbb{R}^{d}$ there exists a control sequence $u \in U^{\mathbb{N}}$ such that $\lim _{t \rightarrow \infty} \Phi_{u}(t, 0) x=0$. System (1) is called (state) feedback stabilizable if there exists a map $F: \mathbb{R}^{d} \rightarrow U$ such that the system

$$
x(t+1)=A(F(x(t))) x(t), \quad t \in \mathbb{N},
$$

is globally asymptotically stable. If $F$ can be chosen such that (3) is exponentially stable, then we call (1) exponentially (state) feedback stabilizable and $F$ is called exponentially stabilizing.

Recall that a nonlinear system is called forward accessible, if for each point the interior of the forward orbit is nonempty. The following theorem states the main result on exponential stabilizability of (1).

Theor em 1. If (2) is forward accessible, then the following statements are equivalent.

(i) System (1) is asymptotically null controllable.

(ii) System (1) is feedback stabilizable with a piecewise constant feedback $F$.

(iii) System (1) is exponentially feedback stabilizable with a piecewise constant feedback $F$.

\section{Construction of the feedback}

In this section we will give a constructive approach for the calculation of the exponentially stabilizing feedback for system (1). For this purpose we will base our construction on a dynamic programming technique using the optimal value function of a discounted optimal control problem. Note that this procedure yields an existence result for stabilizing feedbacks and in addition makes the problem numerically feasible, cp. Remark 3.

From the application point of view one reason why feedback stabilization is preferred to open loop asymptotic null controllability lies in the fact that one expects robustness of the closed loop system against small perturbations. 
Since in this paper we follow an optimal control approach the resulting feedback will in general be discontinuous, and the desired robustness property cannot be obtained as a simple conclusion from the continuous dependency on the initial value. However, the continuity of the associated value function may be used in order to obtain this property.

The construction of the feedback is related to the following optimal control problem: Define the function $q: \mathbb{P}^{d-1} \times U \rightarrow \mathbb{R} \cup\{-\infty\}$ by

$$
q(\xi, u):=\left\{\begin{array}{cl}
\log \frac{\|A(u) x\|}{\|x\|}, & u \in U(\xi) \\
-\infty, & \text { else }
\end{array}\right.
$$

and for $\delta>0$ the $\delta$-discounted cost $J_{\delta}: \mathbb{P}^{d-1} \times U^{\mathbb{N}} \rightarrow \mathbb{R} \cup\{-\infty\}$ defined by

$$
J_{\delta}(\xi, u):=\left\{\begin{array}{cl}
\delta \sum_{s=0}^{t-1} e^{-\delta s} q(\varphi(s ; \xi, u), u(s)), & u \in U^{\mathbb{N}}(\xi) \\
-\infty & \text { else. }
\end{array}\right.
$$

The associated value function on projective space is given by $v_{\delta}(\xi):=\inf _{u \in U} \mathbb{N} J_{\delta}(\xi, u)$. For small $\delta$ the function $v_{\delta}$ may be interpreted as an approximation of the smallest exponential growth rate that may be attained via an arbitrary control sequence from $\xi$. Similar to [1] — where continuous time systems are discussed — our construction of the feedback is now based on this approximating property. Define $F_{\delta}: \mathbb{P}^{d-1} \rightarrow U$ by the following procedure : For any point $\xi \in \mathbb{P}^{d-1}$ such that $v_{\delta}(\xi)>-\infty$ choose a value $u \in U$ such that

$$
q(\xi, u)+e^{-\delta} v_{\delta}(\varphi(1 ; \xi, u))
$$

becomes minimal. To discuss the case $v_{\delta}(\xi)=-\infty$ note that this is only possible if $U \neq U_{\text {inv }}:=\{u \in U \mid \operatorname{det}(A(u)) \neq$ $0\}$. By assumption we may choose an increasing sequence of compact subsets $U_{n} \subset U_{\text {inv }}$ with nonvoid interior, such that $U=\operatorname{cl} \bigcup_{n=1}^{\infty} U_{n}$ and such that system (2) with control range restricted to $U_{n}$ is forward accessible. Denote by $q_{n}, J_{\delta, n}, v_{\delta, n}$ the function defined by (4), (5) with control range restricted to $U_{n}$. If $v_{\delta}(\xi)=-\infty$ choose a sequence $\left\{u_{n}\right\}$ such that for each $n \in \mathbb{N}$

$$
q_{n}\left(\xi, u_{n}\right)+e^{-\delta} v_{\delta, n}\left(\varphi\left(1 ; \xi, u_{n}\right)\right)
$$

becomes minimal and choose $u$ to be a limit point of the sequence $\left\{u_{n}\right\}$.

Define a feedback $F_{\delta}(\xi):=u$ with the choice of $u$ as described above. It may be shown that this feedback law is indeed an optimal control strategy for $v_{\delta}$. Furthermore it holds that

Theorem 2. The following statements are equivalent:

(i) System (1) is feedback stabilizable with a piecewise constant feedback F.

(ii) There exists a $\bar{\delta}>0$ such that for all $0<\delta<\bar{\delta}$ the feedback $F_{\delta}$ is exponentially stabilizing.

Rem a r k 3. By a standard dynamic programming argument the optimal value function $v_{\delta}$ can be characterized as the solution of a discrete Hamilton-Jacobi-Bellman equation. Using the results from [D] the feedback F can be calculated numerically by means of a numerical approximation of $v_{\delta}$. For this numerical treatment it is advantegeous to use one of the approximations $U_{n}$ that were described above, in order to avoid possible problems caused by singularity.

\section{References}

1 GRÜNE, L.: Discrete Feedback Stabilization of Semilinear Control Systems, ESAIM: Control, Optimisation and Calculus of Variations, 1(1996), 207-224

2 GRÜNE, L.: An adaptive grid scheme for the discrete Hamilton-Jacobi-Bellman equation, Numer. Math. 75(1997), 319-337

3 GRÜNE, L., WIRTh, F.: Feedback stabilization of discrete-time semi-linear systems, Technical report, University of Augsburg, (1997).

4 WiRTh, F.: Dynamics of time-varying discrete-time linear systems: Spectral theory and the projected system. SIAM J. Contr. \& Opt., 36(2) (1998) to appear.

Addresses: Dr. Lars GrünE, Institut für Mathematik, Universität Augsburg, 86135 Augsburg, Germany Dr. Fabian Wirth, Institut für Dynamische Systeme, Universität Bremen, 28334 Bremen, Germany 